\title{
Comparison of the Efficacy of Different Drugs on Non-Motor Symptoms of Parkinson's Disease: a Network Meta- Analysis
}

\author{
Bao-Dong Lia Jing-Jun Cui ${ }^{a} \quad$ Jia Songa ${ }^{a} \quad$ Ce Qia Pei-Feng Ma ${ }^{a} \quad$ Ya-Rong Wang ${ }^{a}$ \\ Jing Baib, \\ aDepartment of Neurology, Hebei Province Cangzhou Hospital of Integrated Traditional and Western \\ Medicine, Cangzhou, bDongzhimen Hospital, Beijing University of Chinese Medicine, Beijing, China
}

\section{Key Words}

Parkinson's disease $\cdot$ Non-motor symptoms $\cdot$ UPDRS • Apomorphine $•$ Efficacy $•$ Drug therapy

- Randomized controlled trials $\cdot$ Bayesian network model

\begin{abstract}
Background/Aims: A network meta-analysis is used to compare the efficacy of ropinirole, rasagiline, rotigotine, entacapone, apomorphine, pramipexole, sumanirole, bromocriptine, piribedil and levodopa, with placebo as a control, for non-motor symptoms in Parkinson's disease (PD). Methods: PubMed, Embase and the Cochrane Library were searched from their establishment dates up to January 2017 for randomized controlled trials (RCTs) investigating the efficacy of the above ten drugs on the non-motor symptoms of PD. A network meta-analysis combined the evidence from direct comparisons and indirect comparisons and evaluated the pooled weighted mean difference (WMD) values and surfaces under the cumulative ranking curves (SUCRA). The network meta-analysis included 21 RCTs. Results: The analysis results indicated that, using the United Parkinson's Disease Rating Scale (UPDRS) III, the efficacies of placebo, ropinirole, rasagiline, rotigotine, entacapone, pramipexole, sumanirole and levodopa in treating PD were lower than that of apomorphine (WMD $=-10.90,95 \% \mathrm{CI}=-16.12 \sim-5.48$; WMD $=-11.85,95 \% \mathrm{CI}=-17.31 \sim-6.16 ; \mathrm{WMD}=-11.15,95 \% \mathrm{CI}=-16.64 \sim-5.04 ; \mathrm{WMD}=$ $-11.70,95 \% \mathrm{CI}=-16.98 \sim-5.60 ; \mathrm{WMD}=-11.04,95 \% \mathrm{CI}=-16.97 \sim-5.34 ; \mathrm{WMD}=-13.27,95 \% \mathrm{CI}$ $=-19.22 \sim-7.40 ; \mathrm{WMD}=-10.25,95 \% \mathrm{CI}=-15.66 \sim-4.32 ;$ and $\mathrm{WMD}=-11.60,95 \% \mathrm{CI}=-17.89 \sim-$ 5.57 , respectively). Treatment with ropinirole, rasagiline, rotigotine, entacapone, pramipexole, sumanirole, bromocriptine, piribedil or levodopa, with placebo as a control, on PD exhibited no significant differences on PD symptoms when the UPDRS II was used for evaluation. Moreover, using the UPDRS III, the SUCRA values indicated that a pomorphine had the best efficacy on the non-motor symptoms of PD (99.0\%). Using the UPDRS II, the SUCRA values for ropinirole, rasagiline, rotigotine, entacapone, pramipexole, sumanirole, bromocriptine, piribedil and
\end{abstract}

B. D. Li and J. J. Cui contributed equally to this work.

Dr. Jing Bai

Dongzhimen Hospital, Beijing University of Chinese Medicine,

No. 5, Haiyuncang, Dongcheng District, Beijing 100700 (China)

Tel. +86-010-84013229, E-Mail jingjj_bb@163.com 
levodopa treatments, with placebo as a control, indicated that bromocriptine showed the best efficacy on the non-motor symptoms of PD (75.6\%). Conclusion: Among ropinirole, rasagiline, rotigotine, entacapone, apomorphine, pramipexole, sumanirole, bromocriptine, piribedil and levodopa, with placebo as a control, apomorphine may be the most efficacious drug for therapy in treating the non-motor symptoms of PD.

(C) 2018 The Author(s)

Published by S. Karger AG, Basel

\section{Introduction}

Parkinson's disease (PD) ranks as the second most common neurodegenerative disease, second only to Alzheimer's disease. Its prevalence rate is approximately $0.3 \%$, which increases to $1 \%$ in individuals over 60 years old $[1,2]$. The clinical manifestations of PD include not only the main motor symptoms of resting tremor and slowness but also the non-motor symptoms of hyposmia, cognitive impairment and sleep disorders [3-6]. The pathogenesis of PD is not entirely clear, but it is thought to be caused by aging, genetic predisposition and exposure to environmental toxins [7]. Interestingly, the degeneration or death of substantia nigra-striatum dopamine neurons and development of eosinophilic inclusion bodies commonly occur in PD [8,9]. At present, the treatment and management for PD involves drug therapy, surgery, cell transplantation and rehabilitation [10].

Currently, the clinical therapeutic drugs for PD are divided into symptomatic therapeutic drugs and a variety of targeted therapeutic drugs. The symptomatic therapeutic drugs mainly include levodopa (LD), a dopamine (DA) receptor agonist, monoamine oxidase (MAO-B) inhibitor and catechol-Omethyl transferase (COMT) inhibitor. LD is the most effective oral drug for PD and can complement a multi-DA neurotransmitter, reduce tremor and effectively improve slowness and stiffness [11]. DA receptors such as G protein-coupled receptors are widely distributed in body and can be divided into D1 receptors and D2 receptors according to their functional characteristics. They can expand visceral blood vessels and have diuretic and natriuretic effects [12]. MAO-B inhibitors can protect neurons by anti-oxidative stress, inhibiting synuclein aggregation and reducing apoptosis and neurotrophic effects [13, 14]. A COMT inhibitor combined with LD can extend the "on" time and shorten the "off" time [15]. The eleven therapeutic drugs of PD in the study are LD; DA agonists (ropinirole and pramipexole); DA receptor agonists (rotigotine, apomorphine, sumanirole, piribedil and bromocriptine); a COMT inhibitor (entacapone); and a highly active MAO-B inhibitor (rasagiline). Piribedil is a DA is a receptor agonist but has never been reported to perform better than placebo in improving motor disability in early PD patients [16]. It has been reported that patients with PD have been treated with DA agonists in an effort to reduce motor complications as well as depressive symptoms [17]. In 2011, a review suggested that piribedil, pramipexole, rotigotine, ropinirole, pramipexole extended release, pergolide, and cabergoline were efficacious as symptomatic monotherapies for motor symptoms of PD, and ropinirole prolonged release was likely efficacious [18]. There was also a study that summarized medical treatments for the most common motor and non-motor symptoms in PD [19]. In the current study, a network meta-analysis method is used for the quantitative comparison of different drug therapies for the treatment and management of PD to determine the optimal treatment and course of management for PD [20].

\section{Materials and Methods}

\section{Literature search}

PubMed, Embase and the Cochrane Library were searched from their establishment date to January 2017. A manual search was also performed for the reference lists. The search was conducted using keywords combined with free words, and the index words mainly included PD, drug therapy/medication, efficacy/ effectiveness, randomized controlled trial, etc. 


\section{Cellular Physiology Cell Physiol Biochem 2018;45:119-130 \begin{tabular}{l|l|l} 
and Biochemistry Published online: January 15, 2018 & $\begin{array}{l}\text { (c) } 2018 \text { The Author(s). Published by S. Karger AG, Basel } \\
\text { www.karger.com/cpb }\end{array}$ \\
\hline
\end{tabular}}

Li et al.: Efficacy of Different Drugs on PD

Inclusion and exclusion criteria

The inclusion criteria included: (1) study design: randomized controlled trail (RCT); (2) interventions: placebo, ropinirole, rasagiline, rotigotine, entacapone, apomorphine, pramipexole, sumanirole, bromocriptine, piribedil or LD; (3) study subjects: PD patients over 50 years old; and (4) outcome evaluation: United Parkinson's Disease Rating Scale (UPDRS). The exclusion criteria were as follows: (1) PD patients with mental disorders such as confusion, hallucination, delusion, excitation, delirium and abnormal behavior; (2) PD patients undergoing surgery; (3) PD patients with orthostatic hypotension; (4) PD patients with a history of epilepsy or convulsion; (5) PD patients with clinically relevant hepatic, renal or cardiac disorders; (6) PD patients who were treated with of DA receptor agonists or antipsychotic drugs; (7) studies with insufficient data; (8) non-RCTs; (9) duplicated publications; (10) conference reports, system evaluations or summary articles; and (11) non-English publications.

\section{Data extraction and quality assessment}

Two researchers extracted the data from included literature independently according to a unified data collection form. If there were disputes, a number of researchers would discuss to reach a consensus. More than two researchers used the Cochrane Collaboration's tool for assessing risk of bias in the randomized controlled trials [21]. The tool included six domains, namely, random assignment, allocation concealment, blinding, loss of outcome data, choosing the outcome reports and other biases. The evaluation included a judgment of "yes", "no", or "unclear" for each aspect to determine a low, high, or unclear risk of bias, respectively. When one or no aspects are defined as "unclear" or "no," the study has a low risk of bias. When four or more aspects are deemed "unclear" or "no," the study has a high risk of bias. If two or three aspects are deemed "unclear" or "no," the study has a moderate risk of bias [22]. Quality assessment of publication bias was performed by Review Manager 5 (RevMan 5.2.3, Cochrane Collaboration, Oxford, UK).

\section{Statistical method}

Initially, we conducted traditional pairwise meta-analyses for studies directly comparing various treatment arms. The pooled estimates of weighted mean differences (WMDs) and 95\% credible intervals (CIs) of UPDRS were calculated. Chi-square tests and I-square tests were employed to examine heterogeneity among the studies [23]. Then, a network diagram was drawn by R 3.2.1 software, in which each node represented an intervention measure, the size of the node represented the sample size, and the thickness of the line between nodes represented the number of included studies. Subsequently, a Bayesian network meta-analysis was carried out to compare different interventions to each other. Each analysis was run depending on a non-informative prior for effective sizes as well as precision. Convergence and lack of autocorrelation were examined and determined following four chains and a 20, 000-simulation burnin phase. Finally, direct probability statements were determined from an additional 50, 000-simulation phase [24]. To interpret WMDs, we computed the probability of each intervention being the most effective or safest treatment according to a Bayesian approach, with the help of probability values summarized as surface under the cumulative ranking curve (SUCRA). A larger the SUCRA value indicated a better rank of the intervention $[25,26]$. All computations were finished by applying an R (V.3.1.2) package gemtc (V.0.6) with the Markov Chain Monte Carlo engine Open BUGS (V.3.4.0).

\section{Results}

\section{Baseline characteristics of included publications}

We retrieved 1, 341 related publications in total, among which 382 duplications, 216 letters or reviews and 181 non-English publications were immediately eliminated. In the remaining 562 publications, 223 non-human publications, 142 publications unrelated to PD, 173 publications unrelated to drug therapy and 3 publications without complete data were eliminated. Finally, 21 RCTs published from 2000 to 2015 were enrolled into this network meta-analysis, including 4, 844 cases of PD patients, most of whom took placebo [27-47] (Fig. 1). Among these 21 RCTs, 16 trials were performed in Caucasians, 5 trials were in Asians, while 18 trials were two-arm, and 3 trials were three-arm. The baseline characteristics of included publications are shown in Table 1 and Table 2, and the Cochrane bias evaluation is shown in Fig. 2. 


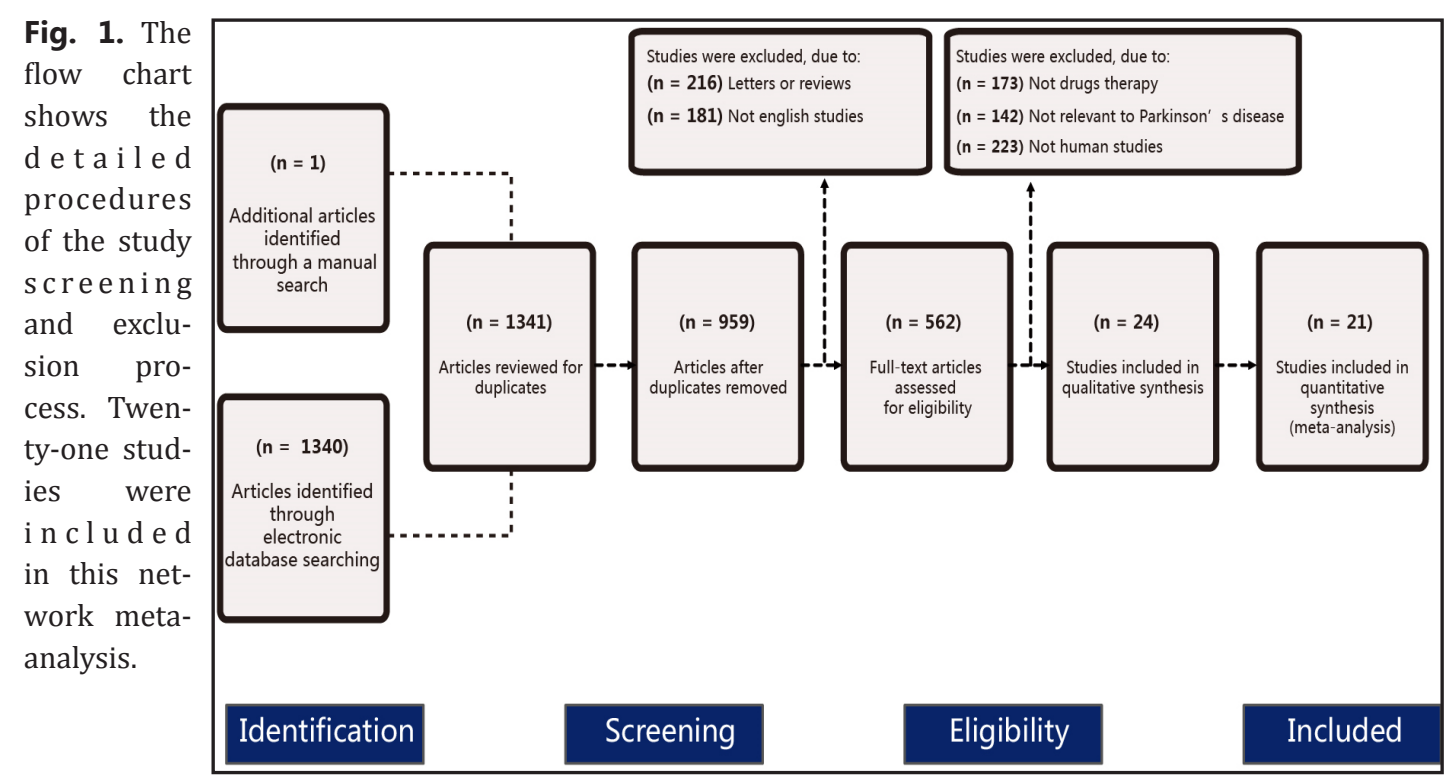

Table 1. The baseline characteristics of included studies. Note: D, drug; M, male; F, female; NR, not report; A = Placebo; $\mathrm{B}=$ Ropinirole; $\mathrm{C}=$ Rasagiline; $\mathrm{D}=$ Rotigotine; $\mathrm{E}=$ Entacapone; $\mathrm{F}=$ Apomorphine; $\mathrm{G}=$ Pramipexole; H = Sumanirole; $\mathrm{I}$ = Bromocriptine; $\mathrm{J}$ = Piribedil; $\mathrm{K}$ = Levodopa

\begin{tabular}{|c|c|c|c|c|c|c|c|c|c|c|c|c|c|c|c|c|c|c|}
\hline \multirow[b]{2}{*}{ First author } & \multirow[b]{2}{*}{ Year } & \multirow[b]{2}{*}{ Ethnicity } & \multicolumn{4}{|c|}{ Treatments } & \multicolumn{3}{|c|}{ Sample size } & \multicolumn{3}{|c|}{ Disease duration (years) } & \multicolumn{2}{|l|}{ Hoehn and } & \multicolumn{2}{|c|}{ Baseline UPDRS (II/III) } & \multirow{2}{*}{ Treatment duration } & \multirow[b]{2}{*}{ Dose of drug } \\
\hline & & & D1 & D2 & D3 & Total & D1 & D2 & D3 & D1 & D2 & D3 & Yahr stage & D1 & D2 & D3 & & \\
\hline Smith KM [27] & 2015 & Caucasians & $\mathrm{A}$ & $\mathrm{C}$ & - & 192 & 99 & 93 & - & NR & NR & & NR & $6.1 / 15.0$ & $5.9 / 14.6$ & & 36-week & 1 or $2 \mathrm{mg} / \mathrm{d}$ \\
\hline Nomoto M [28] & $\begin{array}{l}20 \\
14\end{array}$ & Asians & A & D & - & 172 & 86 & 76 & - & $5.4 \pm 3.0$ & $7.5 \pm 6.0$ & & II-IV & $10.3 / 26.2$ & $11.8 / 28.1$ & & 19-week & $2-16 \mathrm{mg} / \mathrm{d}$ \\
\hline Nicholas AP [29] & 2014 & Caucasians & $\mathrm{A}$ & D & - & 208 & 101 & 107 & - & $\geq 3$ & $\geq 3$ & & II-IV & $12.8 / 26.1$ & $11.8 / 23.1$ & & 16-week & $4 \mathrm{mg} / \mathrm{d}$ \\
\hline Ray Chaudhuri K [30] & 2013 & Caucasians & $\mathrm{A}$ & D & - & 267 & 89 & 178 & - & NR & NR & & $\mathrm{I}-\mathrm{IV}$ & $\mathrm{NR} / 31.8$ & $\mathrm{NR} / 29.7$ & & 12-week & $2-16 \mathrm{mg} / \mathrm{d}$ \\
\hline Mizuno Y [31] & 2013 & Asians & A & D & - & 176 & 88 & 88 & - & $1.8 \pm 1.9$ & $2.0 \pm 1.8$ & & I-III & $7.4 / 28.2$ & $6.8 / 20.2$ & & 19-week & $2-16 \mathrm{mg} / \mathrm{d}$ \\
\hline Grosset KA-a [32] & 2013 & Caucasians & $\mathrm{A}$ & $\mathrm{F}$ & - & 47 & 15 & 32 & - & 5 & 5 & & II-IV & $\mathrm{NR} / 21.1$ & $\mathrm{NR} / 25.7$ & & 20-week & $1.5-4 \mathrm{mg} / \mathrm{d}$ \\
\hline Grosset KA-b [33] & 2013 & Caucasians & $\mathrm{A}$ & $\mathrm{F}$ & - & 55 & 15 & 40 & - & $11.5 \pm 3.2$ & $12.2 \pm 3.9$ & & II-IV & NR & $\mathrm{NR}$ & & 17-week & $1.5-4.5 \mathrm{mg} / \mathrm{d}$ \\
\hline Stocchi F [34] & 2011 & Caucasians & $\mathrm{A}$ & $\mathrm{c}$ & E & 105 & 37 & 323 & 36 & $10.7 \pm 5.8$ & $10.2 \pm 4.5$ & $9.4 \pm 3.7$ & II-IV & $18.8 / 40.3$ & $17.5 / 38.0$ & $18.3 / 39.4$ & 30-week & $1 / 200 \mathrm{mg} / \mathrm{d}$ \\
\hline Watts RL [35] & 2010 & Caucasians & $\mathrm{B}$ & $\mathrm{K}$ & - & 208 & 104 & 104 & - & $2.7 \pm 2.1$ & $2.7 \pm 2.4$ & & I-III & $8.6 / 19.6$ & $8.2 / 19.4$ & & 104-week & $2-24 / 50-1000 \mathrm{mg} / \mathrm{d}$ \\
\hline Hauser RA [36] & 2010 & Caucasians & $\mathrm{A}$ & G & - & 156 & 50 & 106 & - & $0.8 \pm 1.1$ & $1.1 \pm 1.3$ & & I-III & $7.6 / 22.4$ & $7.9 / 22.6$ & & 18-week & $0.375-4.5 \mathrm{mg} / \mathrm{d}$ \\
\hline Singer C [37] & 2007 & Caucasians & S A & B & $\mathrm{H}$ & 609 & 203 & 2022 & 204 & $1.38 \pm 0.13$ & $1.23 \pm 0.10$ & $1.37 \pm 0.11$ & I-III & $8.5 / 20.4$ & $8.1 / 19.1$ & $9.1 / 21.1$ & 40-week & $0.75-24 / 1-16 \mathrm{mg} / \mathrm{d}$ \\
\hline Mizuno Y [38] & 2007 & Asians & A & B & - & 241 & 120 & 121 & - & $5.5 \pm 4.1$ & $5.5 \pm 3.7$ & & II-IV & $\mathrm{NR} / 24.9$ & $\mathrm{NR} / 23.8$ & & 16-week & $0.75-15 \mathrm{mg} / \mathrm{d}$ \\
\hline
\end{tabular}

Table 2. The baseline characteristics of included studies. Note: D, drug; M, male; F, female; NR, not report; A = Placebo; $\mathrm{B}=$ Ropinirole; $\mathrm{C}=$ Rasagiline; $\mathrm{D}=$ Rotigotine; $\mathrm{E}=$ Entacapone; $\mathrm{F}=$ Apomorphine; $\mathrm{G}=$ Pramipexole; $\mathrm{H}$ = Sumanirole; $\mathrm{I}$ = Bromocriptine; $\mathrm{J}$ = Piribedil; $\mathrm{K}$ = Levodopa

\begin{tabular}{|c|c|c|c|c|c|c|c|c|c|c|c|c|c|c|c|c|c|c|}
\hline \multirow{2}{*}{ First author } & \multirow{2}{*}{ Year } & \multirow{2}{*}{ Ethnicity } & \multicolumn{3}{|c|}{ Treatments } & \multirow{2}{*}{ Total } & \multicolumn{3}{|c|}{ Sample size } & \multicolumn{3}{|c|}{ Disease duration (years) } & \multirow{2}{*}{$\begin{array}{l}\text { Hoehn and } \\
\text { Yahr stage }\end{array}$} & \multicolumn{3}{|c|}{ Baseline UPDRS (II/III) } & \multirow{2}{*}{ Treatment duration } & \multirow{2}{*}{ Dose of drug } \\
\hline & & & D1 & D2 & D3 & & D1 & D2 D & D3 & D1 & D2 & D3 & & D1 & D2 & D3 & & \\
\hline Jankovic J [39] & 2007 & Caucasians & A & D & - & 277 & 96 & 181 & - & 5 & 5 & & I-III & $8.7 / 21.3$ & $8.3 / 21.6$ & & 32-week & 2,4, or $6 \mathrm{mg} / \mathrm{d}$ \\
\hline Castro-Caldas A [40] & 2006 & Caucasians & I & $\mathrm{J}$ & - & 425 & 215 & 210 & - & $3.3 \pm 2.4$ & $3.1 \pm 2.0$ & & I-III & $9.2 / 24.1$ & $9.3 / 23.8$ & & 48-week & $1.25-25 / 50-150 \mathrm{mg} / \mathrm{d}$ \\
\hline Reichmann H [41] & 2005 & Caucasians & A & E & - & 270 & 96 & 174 & - & $7.1 \pm 4.0$ & $7.5 \pm 4.7$ & & I-IV & $24.7 / 38.6$ & $23.5 / 37.6$ & & 13-week & $200 \mathrm{mg} / \mathrm{d}$ \\
\hline Rascol O [42] & 2005 & Caucasians & A & c & $\mathrm{E}$ & 687 & 2292 & 23122 & 227 & $8.8 \pm 4.8$ & $8.7 \pm 4.9$ & $9.2 \pm 4.7$ & $\mathrm{NR}$ & $18.9 / 23.7$ & $19.0 / 24.0$ & 19.1/23.1 & 18-week & $1 / 200 \mathrm{mg} / \mathrm{d}$ \\
\hline Wong KS [43] & 2003 & Asians & A & G & - & 150 & 77 & 73 & - & $4.3 \pm 0.4$ & $4.5 \pm 0.4$ & & $\mathrm{I}-\mathrm{IV}$ & $10.8 / 26.6$ & $10.7 / 26.7$ & & 15 -week & $0.375-4.5 \mathrm{mg} / \mathrm{d}$ \\
\hline $\operatorname{Im~JH~[44]~}$ & 2003 & Asians & B & I & - & 76 & 37 & 39 & - & $6.8 \pm 3.8$ & $6.4 \pm 3.2$ & & II-IV & $\mathrm{NR} / 19$ & $\mathrm{NR} / 21.8$ & & 16-week & $0.75-9.0 / 1.25-17.5 \mathrm{mg} / \mathrm{d}$ \\
\hline Brooks DJ [45] & 2003 & Caucasians & A & $\mathrm{E}$ & - & 172 & 571 & 115 & - & $9.1 \pm 5.3$ & $9.6 \pm 5.1$ & & $\mathrm{NR}$ & $9.4 / 22.4$ & $10.3 / 23.4$ & & 24-week & $200 \mathrm{mg} / \mathrm{d}$ \\
\hline Pogarell o [46] & 2002 & Caucasians & A & G & - & 83 & 39 & 44 & - & $6.0 \pm 3.5$ & $6.5 \pm 4.0$ & & $\mathrm{I}-\mathrm{IV}$ & $11.5 / 32.1$ & $13.0 / 34.2$ & & 12-week & $0.375-4.5 \mathrm{mg} / \mathrm{d}$ \\
\hline Rascol O [47] & 2000 & Caucasians & B & $\mathrm{K}$ & - & 268 & 179 & 89 & - & $2.5 \pm 2.8$ & $2.4 \pm 2.3$ & & I-III & $8.0 / 21.5$ & $8.0 / 21.7$ & & NR & $0.75-24 / 50-1200 \mathrm{mg} / \mathrm{d}$ \\
\hline
\end{tabular}


Fig. 2. Cochrane system bias evaluations for each of included publications.

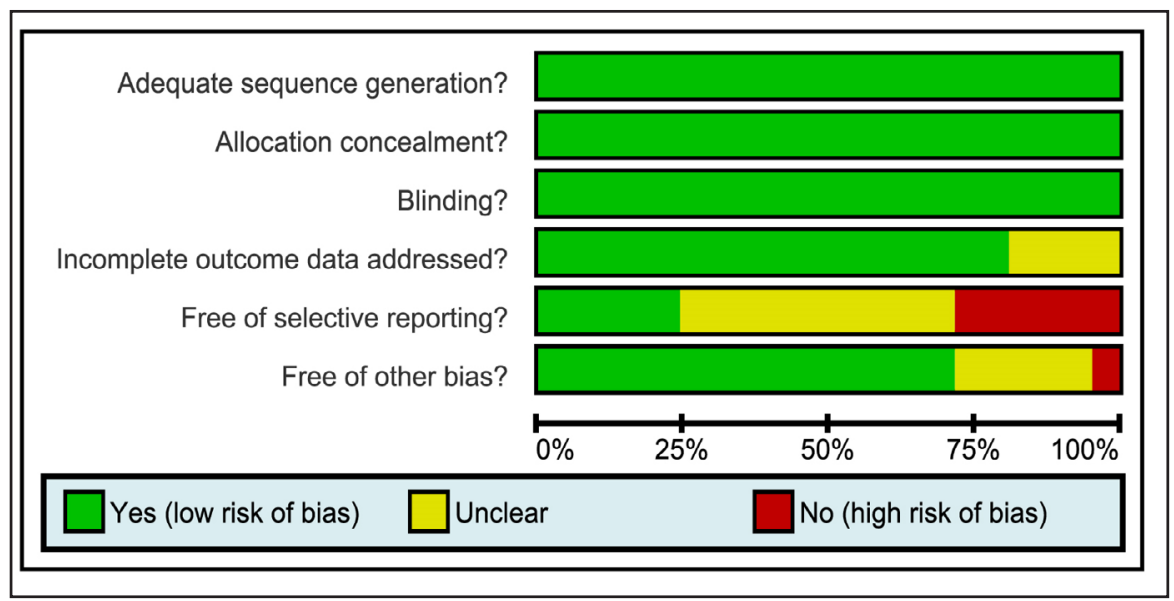

Pairwise meta-analysis of the efficacies of ten drugs on PD

As in shown in Table 3, direct pairwise comparison of the efficacies of ten drugs for PD found that, according to the UPDRS III, the efficacy of placebo in treating PD was worse than those of apomorphine and sumanirole $(\mathrm{WMD}=-10.37,95 \% \mathrm{CI}=$ $-15.72 \sim-5.02$; and $\mathrm{WMD}=-0.60,95 \% \mathrm{CI}=$ $-0.66 \sim-0.54$, respectively) but was better than that of ropinirole (WMD $=0.40,95 \% \mathrm{CI}$ $=0.34 \sim 0.46$ ). Compared with sumanirole, ropinirole exhibited lower efficacy (WMD $=-1.00,95 \% \mathrm{CI}=-1.06 \sim-0.94)$. Using the UPDRS II, placebo and ropinirole showed lower efficacy than sumanirole $(\mathrm{WMD}=-0.60$, $95 \% \mathrm{CI}=-0.66 \sim-0.54$; and $\mathrm{WMD}=-1.00,95 \%$ $\mathrm{CI}=-1.06 \sim-0.94$, respectively), but placebo had a better efficacy than ropinirole (WMD = $0.40,95 \% \mathrm{CI}=0.34 \sim 0.46$ ).

\section{Evidence of a network relationship}

Using the UPDRS III and UPDRS II, we found that most PD patients took placebo, and the majority of direct pairwise comparisons refer to placebo vs. rasagiline, placebo vs. rotigotine, placebo vs. entacapone and placebo vs. pramipexole (Fig. 3).

The main results of the network meta-analysis of the efficacies of ten drugs on PD

The main results of the network meta-analysis showed that, using the UPDRS III, apomorphine presented better efficacy than placebo, ropinirole, rasagiline, rotigotine, entacapone, pramipexole, sumanirole and LD (WMD $=-10.90 ; 95 \% \mathrm{CI}=-16.12 \sim-5.48$; WMD $=-11.85 ; 95 \% \mathrm{CI}=-17.31 \sim-6.16 ; \mathrm{WMD}=-11.15,95 \% \mathrm{CI}=-16.64 \sim-5.04 ; \mathrm{WMD}=-11.70$, $95 \% \mathrm{CI}=-16.98 \sim-5.60 ; \mathrm{WMD}=-11.04,95 \% \mathrm{CI}=-16.97 \sim-5.34 ; \mathrm{WMD}=-13.27,95 \% \mathrm{CI}=$ $-19.22 \sim-7.40$; $\mathrm{WMD}=-10.25,95 \% \mathrm{CI}=-15.66 \sim-4.32$; and $\mathrm{WMD}=-11.60,95 \% \mathrm{CI}=-17.89 \sim-$ 5.57, respectively) Table 4 and Fig. 4). However, when using the UPDRS II, the treatment of PD of using ropinirole, rasagiline, rotigotine, entacapone, pramipexole, sumanirole, bromocriptine, piribedil and LD, with placebo as a control, showed no significant differences in efficacy (Table 5).

Table 3. Pairwise meta-analysis of based on Unified Parkinson's Disease Rating Scale (UPDRS) III UPDRS, Unified Parkinson's Disease Rating Scale; $\mathrm{A}=$ Placebo; $\mathrm{B}=$ Ropinirole; $\mathrm{C}=$ Rasagiline; $\mathrm{D}=$ Pramipexole; $\mathrm{H}=$ Sumanirole; $\mathrm{I}=$ Bromocriptine; $\mathrm{J}$ = Piribedil; $\mathrm{K}$ = Levodopa

\begin{tabular}{|c|c|c|c|c|}
\hline \multirow{2}{*}{ Included studies } & \multirow{2}{*}{ Comparisons } & \multicolumn{3}{|c|}{ Pairwise meta-analysis } \\
\hline & & WMD $(95 \% \mathrm{Cl})$ & & $\mathrm{P}$ \\
\hline $\begin{array}{l}\text { UPDRS III } \\
1 \text { study }\end{array}$ & & & & \\
\hline 3 studies & $\begin{array}{l}\text { Avs. C } \\
\text { Avs. }\end{array}$ & $0.13(-0.60 \sim 0.87)$ & $0.00 \%$ & $\begin{array}{l}\text { NA } \\
0.766\end{array}$ \\
\hline 5 studies & Avs. D & $2.48(-2.14 \sim 7.11)$ & $96.20 \%$ & $<0.001$ \\
\hline 3 studies & A vs. E & $-0.81(-2.07 \sim 0.44)$ & $0.00 \%$ & 0.774 \\
\hline 2 studies & Avs. F & $-10.37(-15.72 \sim 5.02)$ & $0.00 \%$ & 0.691 \\
\hline 3 studies & A vs. G & $4.00(-4.36 \sim 12.36)$ & $84.70 \%$ & 0.001 \\
\hline 1 study & A vs. $\mathrm{H}$ & $-0.60(-0.66 \sim-0.54)$ & NA & NA \\
\hline 1 study & B vs. H & $-1.00(-1.06 \sim-0.94)$ & $\mathrm{NA}$ & $\mathrm{NA}$ \\
\hline 1 study & B vs. I & $-4.20(-9.17 \sim 0.77)$ & NA & $\mathrm{NA}$ \\
\hline 2 studies & B vs. K & $0.28(-1.00 \sim 1.56)$ & $0.00 \%$ & 0.709 \\
\hline 2 studies & Cvs. E & $-0.89(-2.19 \sim 0.42)$ & $0.00 \%$ & 0.958 \\
\hline $\begin{array}{l}1 \text { study } \\
\text { UPDRS II }\end{array}$ & I vs.J & $0.50(-0.42 \sim 1.42)$ & $\mathrm{NA}$ & NA \\
\hline 1 study & A vs. B & $0.40(0.34 \sim 0.46)$ & $\mathrm{NA}$ & NA \\
\hline 3 studies & Avs.C & $0.13(-0.60 \sim 0.87)$ & $0.00 \%$ & 0.766 \\
\hline 3 studies & Avs. D & $3.20(-3.64 \sim 10.03)$ & $98.00 \%$ & $<0.001$ \\
\hline 4 studies & A vs. E & $0.33(-1.69 \sim 2.35)$ & $45.20 \%$ & 0.088 \\
\hline 3 studies & A vs. G & $4.00(-4.36 \sim 12.36)$ & $84.70 \%$ & 0.001 \\
\hline 1 study & A vs. H & $-0.60(-0.66 \sim-0.54)$ & $\mathrm{NA}$ & $\mathrm{NA}$ \\
\hline 1 study & B vs. H & $-1.00(-1.06 \sim-0.94)$ & $\mathrm{NA}$ & $\mathrm{NA}$ \\
\hline 1 study & Bvs.I & $-4.20(-9.17 \sim 0.77)$ & $\mathrm{NA}$ & NA \\
\hline 2 studies & B vs. K & $0.17(-0.47 \sim 0.80)$ & $0.00 \%$ & 0.95 \\
\hline 2 studies & Cvs. E & $1.61(-2.79 \sim 6.02)$ & $96.80 \%$ & $<0.001$ \\
\hline 1 study & I vs.J & $0.50(-0.42 \sim 1.42)$ & $\mathrm{NA}$ & NA \\
\hline
\end{tabular}


Cumulative probability of sorting

Using the UPDRS III, the SUCRA values for ropinirole, rasagiline, rotigotine, entacapone, apomorphine, pramipexole, sumanirole, bromocriptine, piribedil and LD, with placebo as a control, showed that apomorphine exhibited the best efficacy $(90 \%)$, but pramipexole presented the worst efficacy (8.7\%) (Fig. 5A). Using the UPDRS II, the SUCRA values for ropinirole, rasagiline, rotigotine, entacapone, pramipexole, sumanirole, bromocriptine, piribedil and LD, with placebo as a control, demonstrated that the efficacy of bromocriptine on PD was the best (75.6\%), while the efficacy of rotigotine on PD was the worst (22.0\%) (Fig. 5B).

\section{Discussion}

The network meta-analysis method was used to evaluate differences in efficacy for ropinirole, rasagiline, rotigotine, entacapone, apomorphine, pramipexole, sumanirole, bromocriptine, pPiribedil and LD, with placebo as a control, in the treatment and management of PD.

The direct comparison of pairwise meta-analysis showed that the efficacy of apomorphine and sumanirole for treating PD were better than other drugs. The probable reason is that the main pathological changes of PD patients are the degeneration or loss of neuronal tissue and the decrease of striatal DA neurotransmitter. Both apomorphine and sumanirole are DA receptor activators. They have a long half-life and can remain in the blood to improve the dyskinesia induced by long-term use of LD and reduce the severity of the "on-off" effect [48]. Moreover, the DA receptor activators apomorphine and sumanirole can stimulate the viable DA receptors to

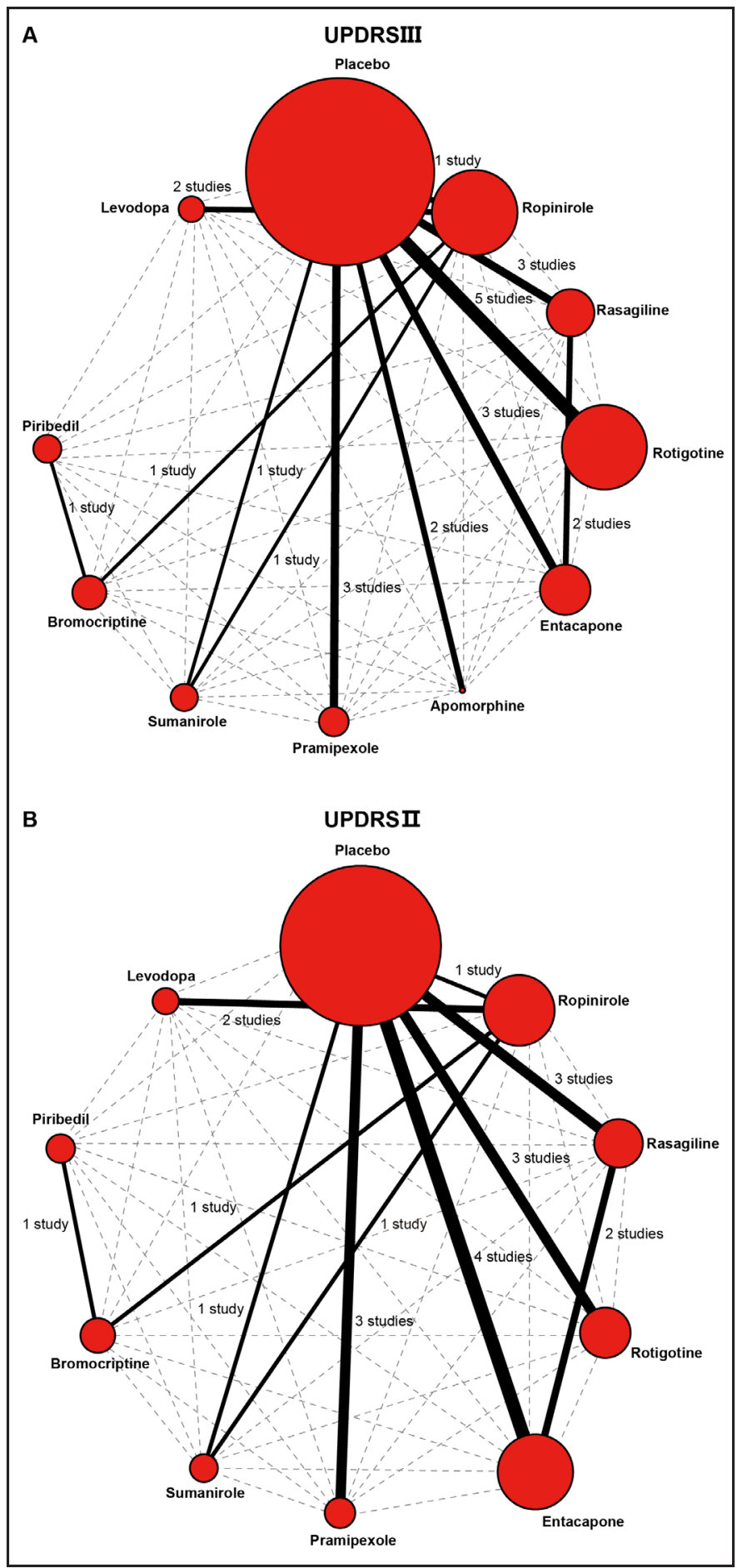

Fig. 3. Network evidence diagrams of the efficacy of ten drugs for Parkinson's disease using the United Parkinson's Disease Rating Scale (UPDRS) III and the UPDRS II of Parkinson's disease. (Note: A = UPDRS III; B = UPDRS II).

\section{KARGER}


Table 4. Primary outcomes of UPDRSIII improvements. Notes: UPDRS, Unified Parkinson's Disease Rating Scale; bold numbers represent the differences are of significances

\begin{tabular}{|c|c|c|c|c|c|c|c|c|c|c|}
\hline Placebo & $\begin{array}{c}-0.91 \\
(-2.88,1.00)\end{array}$ & $\begin{array}{c}-0.25 \\
(-2.17,1.71)\end{array}$ & $\begin{array}{c}-0.70 \\
(-2.37,0.92)\end{array}$ & $\begin{array}{c}-0.29 \\
(-2.42,2.03)\end{array}$ & $\begin{array}{c}10.90 \\
(5.48,16.12)\end{array}$ & $\begin{array}{c}-2.56 \\
(-5.33,0.43)\end{array}$ & $\begin{array}{c}0.69 \\
(-1.62,3.01)\end{array}$ & $\begin{array}{c}3.72 \\
(-2.17,9.11)\end{array}$ & $\begin{array}{c}3.50 \\
(-3.23,9.52)\end{array}$ & $\begin{array}{c}-0.78 \\
(-4.12,2.24)\end{array}$ \\
\hline $\begin{array}{l}0.91 \\
(-1.00,2.88)\end{array}$ & Ropinirole & $\begin{array}{c}0.64 \\
(-1.95,3.39)\end{array}$ & $\begin{array}{c}0.23 \\
(-2.40,2.73)\end{array}$ & $\begin{array}{c}0.63 \\
(-2.19,3.61)\end{array}$ & $\begin{array}{c}11.85 \\
(6.16,17.31)\end{array}$ & $\begin{array}{c}-1.62 \\
(-4.94,1.92)\end{array}$ & $\begin{array}{c}1.62 \\
(-0.68,3.93)\end{array}$ & $\begin{array}{c}4.59 \\
(-0.91,9.95)\end{array}$ & $\begin{array}{c}4.41 \\
(-1.97,10.31)\end{array}$ & $\begin{array}{c}0.13 \\
(-2.50,2.65)\end{array}$ \\
\hline $\begin{array}{l}0.25 \\
(-1.71,2.17)\end{array}$ & $\begin{array}{c}-0.64 \\
(-3.39,1.95)\end{array}$ & Rasagiline & $\begin{array}{c}-0.50 \\
(-2.96,2.09)\end{array}$ & $\begin{array}{c}-0.02 \\
(-2.41,2.37)\end{array}$ & $\begin{array}{c}11.15 \\
(5.04,16.64)\end{array}$ & $\begin{array}{c}-2.37 \\
(-5.62,1.18)\end{array}$ & $\begin{array}{c}0.96 \\
(-2.00,3.84)\end{array}$ & $\begin{array}{c}3.95 \\
(-2.52,9.71)\end{array}$ & $\begin{array}{c}3.74 \\
(-3.43,10.27)\end{array}$ & $\begin{array}{c}-0.51 \\
(-4.35,2.96)\end{array}$ \\
\hline $\begin{array}{l}0.70 \\
(-0.92,2.37)\end{array}$ & $\begin{array}{c}-0.23 \\
(-2.73,2.40)\end{array}$ & $\begin{array}{c}0.50 \\
(-2.09,2.96)\end{array}$ & Rotigotine & $\begin{array}{c}0.38 \\
(-2.33,3.21)\end{array}$ & $\begin{array}{c}11.70 \\
(5.60,16.98)\end{array}$ & $\begin{array}{c}-1.83 \\
(-5.10,1.62)\end{array}$ & $\begin{array}{c}1.41 \\
(-1.48,4.23)\end{array}$ & $\begin{array}{c}4.41 \\
(-1.88,10.12)\end{array}$ & $\begin{array}{c}4.24 \\
(-2.80,10.45)\end{array}$ & $\begin{array}{c}-0.07 \\
(-3.84,3.46)\end{array}$ \\
\hline $\begin{array}{l}0.29 \\
(-2.03,2.42)\end{array}$ & $\begin{array}{c}-0.63 \\
(-3.61,2.19)\end{array}$ & $\begin{array}{c}0.02 \\
(-2.37,2.41)\end{array}$ & $\begin{array}{c}-0.38 \\
(-3.21,2.33)\end{array}$ & Entacapone & $\begin{array}{c}11.04 \\
(5.34,16.97)\end{array}$ & $\begin{array}{c}-2.21 \\
(-5.77,1.43)\end{array}$ & $\begin{array}{c}1.01 \\
(-2.25,4.03)\end{array}$ & $\begin{array}{c}4.13 \\
(-2.53,9.91)\end{array}$ & $\begin{array}{c}3.95 \\
(-3.49,10.46)\end{array}$ & $\begin{array}{c}-0.53 \\
(-4.59,3.11)\end{array}$ \\
\hline $\begin{array}{l}-10.90 \\
(-16.12,-5.48)\end{array}$ & $\begin{array}{c}-11.85 \\
(-17.31,-6.16)\end{array}$ & $\begin{array}{c}-11.15 \\
(-16.64,-5.04)\end{array}$ & $\begin{array}{c}-11.70 \\
(-16.98,-5.60)\end{array}$ & $\begin{array}{c}-11.04 \\
(-16.97,-5.34)\end{array}$ & Apomorphine & $\begin{array}{c}-13.27 \\
(-19.22,-7.40)\end{array}$ & $\begin{array}{c}-10.25 \\
(-15.66,-4.32)\end{array}$ & $\begin{array}{c}-7.05 \\
(-15.18,1.74)\end{array}$ & $\begin{array}{c}-7.10 \\
(-16.27,1.64)\end{array}$ & $\begin{array}{c}-11.60 \\
(-17.89,-5.57)\end{array}$ \\
\hline $\begin{array}{l}2.56 \\
(-0.43,5.33)\end{array}$ & $\begin{array}{c}1.62 \\
(-1.92,4.94)\end{array}$ & $\begin{array}{c}2.37 \\
(-1.18,5.62)\end{array}$ & $\begin{array}{c}1.83 \\
(-1.62,5.10)\end{array}$ & $\begin{array}{c}2.21 \\
(-1.43,5.77)\end{array}$ & $\begin{array}{c}13.27 \\
(7.40,19.22)\end{array}$ & Pramipexole & $\begin{array}{c}3.25 \\
(-0.49,6.78)\end{array}$ & $\begin{array}{c}6.10 \\
(-0.58,12.91)\end{array}$ & $\begin{array}{c}5.96 \\
(-1.41,13.03)\end{array}$ & $\begin{array}{c}1.80 \\
(-3.00,5.81)\end{array}$ \\
\hline $\begin{array}{l}-0.69 \\
(-3.01,1.62)\end{array}$ & $\begin{array}{c}-1.62 \\
(-3.93,0.68)\end{array}$ & $\begin{array}{c}-0.96 \\
(-3.84,2.00)\end{array}$ & $\begin{array}{c}-1.41 \\
(-4.23,1.48)\end{array}$ & $\begin{array}{c}-1.01 \\
(-4.03,2.25)\end{array}$ & $\begin{array}{c}10.25 \\
(4.32,15.66)\end{array}$ & $\begin{array}{c}-3.25 \\
(-6.78,0.49)\end{array}$ & Sumanirole & $\begin{array}{c}3.01 \\
(-2.99,8.59)\end{array}$ & $\begin{array}{c}2.84 \\
(-4.00,9.02)\end{array}$ & $\begin{array}{c}-1.45 \\
(-5.01,1.76)\end{array}$ \\
\hline $\begin{array}{l}-3.72 \\
(-9.11,2.17)\end{array}$ & $\begin{array}{c}-4.59 \\
(-9.95,0.91)\end{array}$ & $\begin{array}{c}-3.95 \\
(-9.71,2.52)\end{array}$ & $\begin{array}{c}-4.41 \\
(-10.12,1.88)\end{array}$ & $\begin{array}{c}-4.13 \\
(-9.91,2.53)\end{array}$ & $\begin{array}{c}7.05 \\
(-1.74,15.18)\end{array}$ & $\begin{array}{c}-6.10 \\
(-12.91,0.58)\end{array}$ & $\begin{array}{c}-3.01 \\
(-8.59,2.99)\end{array}$ & Bromocriptine & $\begin{array}{c}-0.12 \\
(-3.13,2.83)\end{array}$ & $\begin{array}{c}-4.63 \\
(-10.61,1.63)\end{array}$ \\
\hline $\begin{array}{l}-3.50 \\
(-9.52,3.23)\end{array}$ & $\begin{array}{c}-4.41 \\
(-10.31,1.97)\end{array}$ & $\begin{array}{c}-3.74 \\
(-10.27,3.43)\end{array}$ & $\begin{array}{c}-4.24 \\
(-10.45,2.80)\end{array}$ & $\begin{array}{c}-3.95 \\
(-10.46,3.49)\end{array}$ & $\begin{array}{c}7.10 \\
(-1.64,16.27)\end{array}$ & $\begin{array}{c}-5.96 \\
(-13.03,1.41)\end{array}$ & $\begin{array}{c}-2.84 \\
(-9.02,4.00)\end{array}$ & $\begin{array}{c}0.12 \\
(-2.83,3.13)\end{array}$ & Piribedil & $\begin{array}{c}-4.46 \\
(-10.96,2.55)\end{array}$ \\
\hline $\begin{array}{l}0.78 \\
(-2.24,4.12)\end{array}$ & $\begin{array}{c}-0.13 \\
(-2.65,2.50)\end{array}$ & $\begin{array}{c}0.51 \\
(-2.96,4.35)\end{array}$ & $\begin{array}{c}0.07 \\
(-3.46,3.84)\end{array}$ & $\begin{array}{c}0.53 \\
(-3.11,4.59)\end{array}$ & $\begin{array}{c}11.60 \\
(5.57,17.89)\end{array}$ & $\begin{array}{c}-1.80 \\
(-5.81,3.00)\end{array}$ & $\begin{array}{c}1.45 \\
(-1.76,5.01)\end{array}$ & $\begin{array}{c}4.63 \\
(-1.63,10.61)\end{array}$ & $\begin{array}{c}4.46 \\
(-2.55,10.96)\end{array}$ & Levodopa \\
\hline
\end{tabular}

Fig. 4. The relative relationship forest plots of the efficacy of ten drugs for Parkinson's disease using the United Parkinson's Disease Rating Scale (UPDRS) III. (Note: $\mathrm{A}=$ placebo; $\mathrm{B}=$ ropinirole; $\mathrm{C}=$ rasagiline; $\mathrm{D}=$ rotigotine; $\mathrm{E}=\mathrm{en}$ tacapone; $\mathrm{F}=$ apomorphine; $\mathrm{G}=$ pramipexole; $\mathrm{H}$ = sumanirole; $\mathrm{I}=$ bromocriptine; $\mathrm{J}=$ piribedil; $\mathrm{K}=$ levodopa).

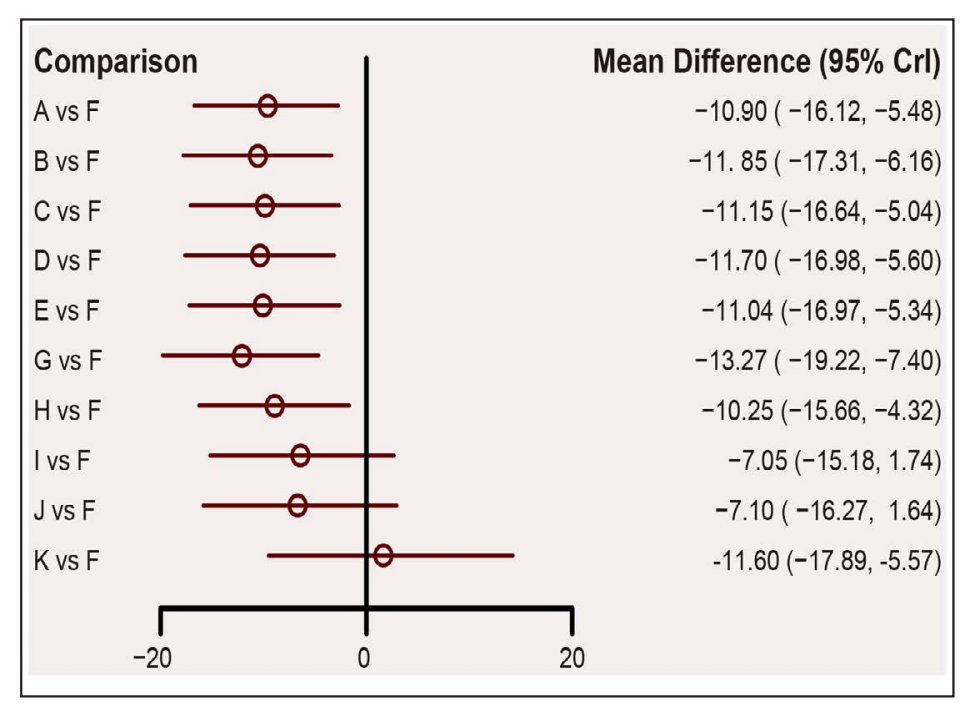

down-regulate the expression of the apoptotic protein Bax and up-regulate the expression of the anti-apoptosis protein Bcl-2 [49]. This will indirectly increase the number of neurons, inhibit the abnormal accumulation caused by amyloid filaments of $\alpha$-synuclein conformational change, delay the development of early symptoms of PD and improve depression symptoms of PD patients [50].

The results of this network meta-analysis further showed that, according to analysis using the UPDRS III, the efficacy of apomorphine was the best among the eleven drugs examined. The reasons are as follows: First, apomorphine can improve the number and activity of DA neurons, which will alleviate the memory disorder seen in PD patients [51]. Moreover, apomorphine can reduce glucose metabolism in the left frontal region and, at the same time, increase glucose metabolism in right occipital temporal region. This will positively regulate the motor function and mood of PD patients and change the motor, cognitive, and motivational state of brain regions in PD patients to improve their quality of life $[52,53]$. Second, apomorphine may alter the expression of important early PD- 
Table 5. Primary outcomes of UPDRS II improvements. Notes: UPDRS, Unified Parkinson's Disease Rating Scale

\begin{tabular}{|c|c|c|c|c|c|c|c|c|c|}
\hline Placebo & $\begin{array}{c}-0.34 \\
(-8.83,7.77)\end{array}$ & $\begin{array}{c}-0.64 \\
(-5.44,4.17)\end{array}$ & $\begin{array}{c}-3.19 \\
(-8.18,1.89)\end{array}$ & $\begin{array}{c}-0.28 \\
(-4.57,4.08)\end{array}$ & $\begin{array}{c}-2.26 \\
(-7.70,2.65)\end{array}$ & $\begin{array}{c}0.60 \\
(-8.07,8.94)\end{array}$ & $\begin{array}{c}3.79 \\
(-8.82,16.71)\end{array}$ & $\begin{array}{c}3.31 \\
(-11.77,19.06)\end{array}$ & $\begin{array}{c}-0.37 \\
(-10.90,9.91)\end{array}$ \\
\hline $\begin{array}{l}0.34 \\
(-7.77,8.83)\end{array}$ & Ropinirole & $\begin{array}{c}-0.34 \\
(-9.70,9.48)\end{array}$ & $\begin{array}{c}-2.82 \\
(-12.31,7.17)\end{array}$ & $\begin{array}{c}0.03 \\
(-9.13,9.58)\end{array}$ & $\begin{array}{c}-1.93 \\
(-11.93,7.88)\end{array}$ & $\begin{array}{c}0.97 \\
(-7.63,9.45)\end{array}$ & $\begin{array}{c}4.17 \\
(-5.53,14.21)\end{array}$ & $\begin{array}{c}3.58 \\
(-9.09,16.80)\end{array}$ & $\begin{array}{c}-0.03 \\
(-6.28,6.06)\end{array}$ \\
\hline $\begin{array}{l}0.64 \\
(-4.17,5.44)\end{array}$ & $\begin{array}{c}0.34 \\
(-9.48,9.70)\end{array}$ & Rasagiline & $\begin{array}{c}-2.55 \\
(-9.60,4.58)\end{array}$ & $\begin{array}{c}0.40 \\
(-5.13,5.57)\end{array}$ & $\begin{array}{c}-1.57 \\
(-8.90,5.13)\end{array}$ & $\begin{array}{c}1.17 \\
(-8.89,11.04)\end{array}$ & $\begin{array}{c}4.47 \\
(-9.15,18.31)\end{array}$ & $\begin{array}{c}3.96 \\
(-12.14,20.47)\end{array}$ & $\begin{array}{c}0.21 \\
(-11.18,11.61)\end{array}$ \\
\hline $\begin{array}{l}3.19 \\
(-1.89,8.18)\end{array}$ & $\begin{array}{c}2.82 \\
(-7.17,12.31)\end{array}$ & $\begin{array}{c}2.55 \\
(-4.58,9.60)\end{array}$ & Rotigotine & $\begin{array}{c}2.88 \\
(-3.69,9.52)\end{array}$ & $\begin{array}{c}0.89 \\
(-6.46,7.87)\end{array}$ & $\begin{array}{c}3.79 \\
(-6.29,13.56)\end{array}$ & $\begin{array}{c}7.00 \\
(-6.68,20.89)\end{array}$ & $\begin{array}{c}6.48 \\
(-9.67,22.52)\end{array}$ & $\begin{array}{c}2.81 \\
(-8.95,13.98)\end{array}$ \\
\hline $\begin{array}{l}0.28 \\
(-4.08,4.57)\end{array}$ & $\begin{array}{c}-0.03 \\
(-9.58,9.13)\end{array}$ & $\begin{array}{c}-0.40 \\
(-5.57,5.13)\end{array}$ & $\begin{array}{c}-2.88 \\
(-9.52,3.69)\end{array}$ & Entacapone & $\begin{array}{c}-2.00 \\
(-8.89,4.58)\end{array}$ & $\begin{array}{c}0.86 \\
(-8.89,10.17)\end{array}$ & $\begin{array}{c}4.05 \\
(-9.10,17.82)\end{array}$ & $\begin{array}{c}3.58 \\
(-12.16,19.57)\end{array}$ & $\begin{array}{c}-0.17 \\
(-11.65,10.76)\end{array}$ \\
\hline $\begin{array}{l}2.26 \\
(-2.65,7.70)\end{array}$ & $\begin{array}{c}1.93 \\
(-7.88,11.93)\end{array}$ & $\begin{array}{c}1.57 \\
(-5.13,8.90)\end{array}$ & $\begin{array}{c}-0.89 \\
(-7.87,6.46)\end{array}$ & $\begin{array}{c}2.00 \\
(-4.58,8.89)\end{array}$ & Pramipexole & $\begin{array}{c}2.89 \\
(-6.83,12.74)\end{array}$ & $\begin{array}{c}6.09 \\
(-7.56,20.28)\end{array}$ & $\begin{array}{c}5.46 \\
(-10.47,22.30)\end{array}$ & $\begin{array}{c}1.84 \\
(-9.58,13.62)\end{array}$ \\
\hline $\begin{array}{l}-0.60 \\
(-8.94,8.07)\end{array}$ & $\begin{array}{c}-0.97 \\
(-9.45,7.63)\end{array}$ & $\begin{array}{c}-1.17 \\
(-11.04,8.89)\end{array}$ & $\begin{array}{c}-3.79 \\
(-13.56,6.29)\end{array}$ & $\begin{array}{c}-0.86 \\
(-10.17,8.89)\end{array}$ & $\begin{array}{c}-2.89 \\
(-12.74,6.83)\end{array}$ & Sumanirole & $\begin{array}{c}3.27 \\
(-9.47,16.47)\end{array}$ & $\begin{array}{c}2.74 \\
(-12.52,18.72)\end{array}$ & $\begin{array}{c}-0.99 \\
(-11.54,9.67)\end{array}$ \\
\hline $\begin{array}{l}-3.79 \\
(-16.71,8.82)\end{array}$ & $\begin{array}{c}-4.17 \\
(-14.21,5.53)\end{array}$ & $\begin{array}{c}-4.47 \\
(-18.31,9.15)\end{array}$ & $\begin{array}{c}-7.00 \\
(-20.89,6.68)\end{array}$ & $\begin{array}{c}-4.05 \\
(-17.82,9.10)\end{array}$ & $\begin{array}{c}-6.09 \\
(-20.28,7.56)\end{array}$ & $\begin{array}{c}-3.27 \\
(-16.47,9.47)\end{array}$ & Bromocriptine & $\begin{array}{c}-0.55 \\
(-8.96,8.11)\end{array}$ & $\begin{array}{c}-4.24 \\
(-15.97,7.38)\end{array}$ \\
\hline $\begin{array}{l}-3.31 \\
(-19.06,11.77)\end{array}$ & $\begin{array}{c}-3.58 \\
(-16.80,9.09)\end{array}$ & $\begin{array}{c}-3.96 \\
(-20.47,12.14)\end{array}$ & $\begin{array}{c}-6.48 \\
(-22.52,9.67)\end{array}$ & $\begin{array}{c}-3.58 \\
(-19.57,12.16)\end{array}$ & $\begin{array}{c}-5.46 \\
(-22.30,10.47)\end{array}$ & $\begin{array}{c}-2.74 \\
(-18.72,12.52)\end{array}$ & $\begin{array}{c}0.55 \\
(-8.11,8.96)\end{array}$ & Piribedil & $\begin{array}{c}-3.71 \\
(-18.39,10.87)\end{array}$ \\
\hline $\begin{array}{l}0.37 \\
(-9.91,10.90)\end{array}$ & $\begin{array}{c}0.03 \\
(-6.06,6.28)\end{array}$ & $\begin{array}{c}-0.21 \\
(-11.61,11.18)\end{array}$ & $\begin{array}{c}-2.81 \\
(-13.98,8.95)\end{array}$ & $\begin{array}{c}0.17 \\
(-10.76,11.65)\end{array}$ & $\begin{array}{c}-1.84 \\
(-13.62,9.58)\end{array}$ & $\begin{array}{c}0.99 \\
(-9.67,11.54)\end{array}$ & $\begin{array}{c}4.24 \\
(-7.38,15.97)\end{array}$ & $\begin{array}{c}3.71 \\
(-10.87,18.39)\end{array}$ & Levodopa \\
\hline
\end{tabular}

related genes. For example, it can stimulate neurotransmitters or neuromodulators in the brain to act on the Jun protein of the postsynaptic membrane, improve the transcription efficiency of downstream genes by activating the expression of AP-1 and CRE promoting factors, and directly assess the degree of the damage to neurons [54]. Lastly, the increase of Fos protein expression is an important manifestation of neuronal vitality, which can inhibit the increase of tumor necrosis factor, glial-derived neurotrophic factor and cyclinB2 mRNA, further inhibiting striatal neurodegeneration and improving resistance to infection and neurotrophic activity $[55,56]$. However, apomorphine will easily degrade under light, and it can inhibit the central nervous system, which causes persistent vomiting, shortness of breath, respiratory depression, acute circulatory failure, coma, death and eosinophilia. Bradycardia and hypotension can occur 5-10 min after treatment and can even progress to acute circulatory failure $[53,57]$.

The SUCRA values showed that the efficacy of apomorphine was the best of the above ten drugs, which was consistent with the results of the network meta-analysis. Among the remaining nine drugs (ropinirole, rasagiline, rotigotine, entacapone, pramipexole, sumanirole, bromocriptine, piribedil and LD, with placebo as a control), the efficacy of bromocriptine on PD was the best. The probable reason is that bromocriptine, at low doses, can preferentially activate presynaptic DA receptors and combine with the activation and antagonistic parts of DA receptors [58]. Bromocriptine can also activate synaptic auto-receptors to sustain the normal function of the receptors, reducing the volatility of symptoms and improving the exercise capacity of patients with PD [59]. As a short-acting receptor agonist, apomorphine could be improved the activity to induce rotational behavior in rodent under stimulation of Hydrogen sulfide [60].

This study used a Bayesian network model to match the direct evidence and indirect evidence in the 21 RCTs while comparing the efficacy of ten drugs in treating PD. The network meta-analysis is a statistical method that can perform quantitative comparisons of different interventions intended to treat the same disease. Its biggest benefit is that it can also sort comparisons according to indexes to choose the best treatment plan [61]. This study has clinical guiding significance for the treatment of PD. Regarding inclusion criteria, the UPDRS has both strengths and weaknesses; however, a study confirmed the decision to prioritize the UPDRS as the first Task Force assessment [62]. MDS-UPDRS has reported excellent factor validity [63]. However, the MDS-UPDRS takes a long time, which causes difficulty in clinical and research settings [64]. The included articles used the UPDRS as the inclusion criteria, 
except one article using both UPDRS and MDS-UPDRS. Therefore, in this study, we took the UPDRS as our inclusion criteria. The dosages of drugs were as follows: ropinirole was $0.75-24 \mathrm{mg} / \mathrm{d}$, rasagiline was 1 or $2 \mathrm{mg} / \mathrm{d}$, rotigotine was $2-16 \mathrm{mg} / \mathrm{d}$, entacapone was $200 \mathrm{mg} / \mathrm{d}$, apomorphine was $1.5-4.5 \mathrm{mg} / \mathrm{d}$, pramipexole was $0.375-4.5 \mathrm{mg} / \mathrm{d}$, sumanirole was $1-16$ $\mathrm{mg} / \mathrm{d}$, bromocriptine was $1.25-25 \mathrm{mg} / \mathrm{d}$, piribedil was $50-150 \mathrm{mg} / \mathrm{d}$, and LD was $50-1200 \mathrm{mg} / \mathrm{d}$. Drugs in each article were at the same dosages, therefore the dosage should not affect our results. The sample sizes of the ten interventions were different and the number of included pairwise comparisons was different, so those factors could affect the research results. The improvement of the UPDRS III was used to evaluate to the efficacy of ten drugs for treating PD, while the improvement of the UPDRS II was used to evaluate the efficacy of nine drugs for treating PD. The unequal number of interventions prevented cluster analysis, which would have likely resulted in different research results.

Above all, our results showed that, among ropinirole, rasagiline, rotigotine, entacapone, apomorphine, pramipexole, sumanirole, bromocriptine, piribedil and LD, with placebo as a control, apomorphine may be the most efficacious drug therapy for PD. From Table 1 and Table 2, we can see that there are no significant differences in the disease duration, Hoehn \& Yahr scores (disease stage), pretreatment UPDRS, treatment duration or baseline UPDRS (II/III) scores. However, due to a few articles without specific values, we could not do a metaregression or a subgroup analysis. Larger sample sizes are needed to confirm the findings of this meta-analysis.

\section{Acknowledgements}

The authors wish to express their gratitude to reviewers for their critical comments.

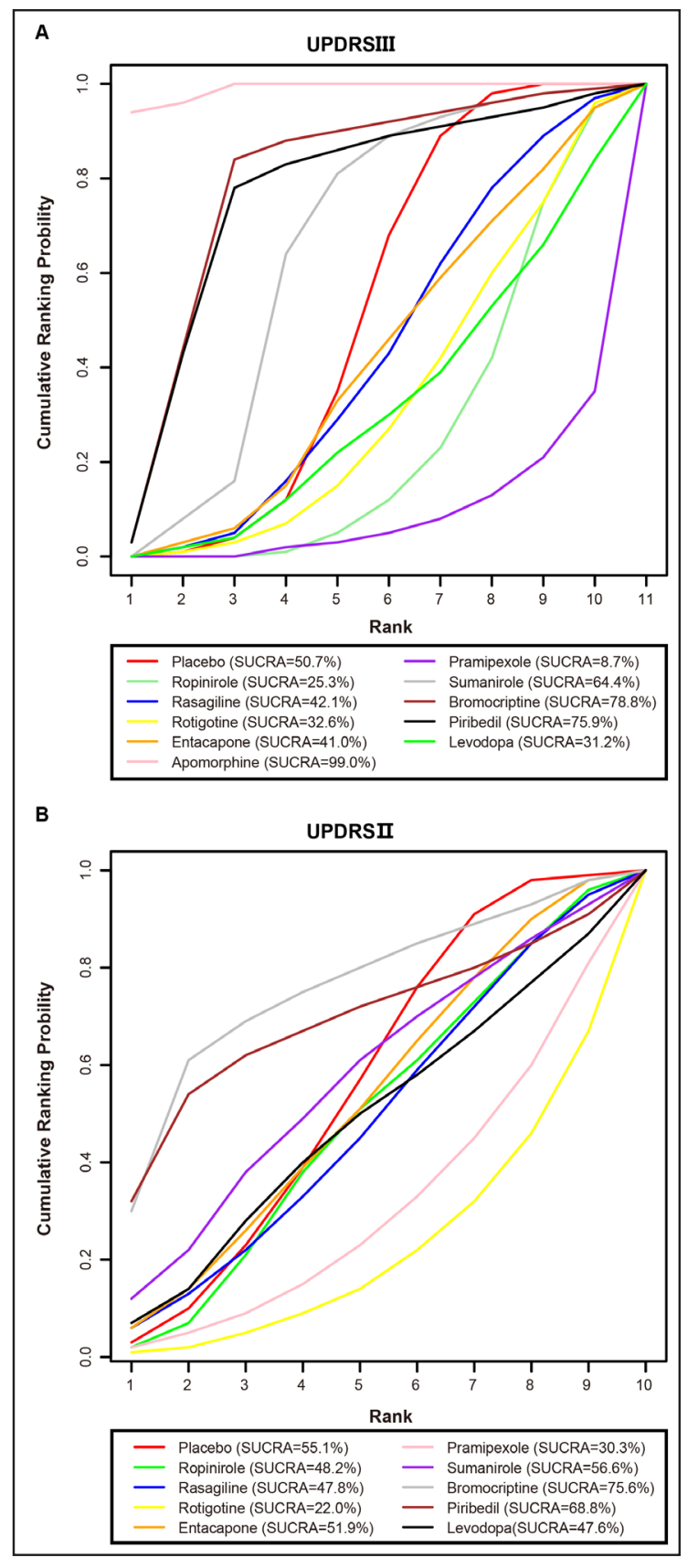

Fig. 5. The cumulative ranking probability diagram of the efficacy of ten drugs for Parkinson's disease using the United Parkinson's Disease Rating Scale (UPDRS) III and the UPDRS II of Parkinson's disease. (Note: A = UPDRS III; B = UPDRS II).

\section{Disclosure Statement}

There are no conflicts of interest to declare for this paper. 


\section{Cellular Physiology Cell Physiol Biochem 2018;45:119-130 \begin{tabular}{ll|l} 
and Biochemistry Published online: January 15, 2018 & $\begin{array}{l}\text { (c) } 2018 \text { The Author(s). Published by S. Karger AG, Basel } \\
\text { www.karger.com/cpb }\end{array}$ \\
\hline
\end{tabular}}

Li et al.: Efficacy of Different Drugs on PD

\section{References}

1 de Lau LM, Breteler MM: Epidemiology of Parkinson's disease. Lancet Neurol 2006;5:525-535.

2 Delenclos M, Jones DR, McLean PJ, Uitti RJ: Biomarkers in Parkinson's disease: Advances and strategies. Parkinsonism Relat Disord 2016;22:S106-110.

3 Martinez-Martin P, Rodriguez-Blazquez C, Kurtis MM, Chaudhuri KR, Group NV: The impact of non-motor symptoms on health-related quality of life of patients with Parkinson's disease. Mov Disord 2011;26:399406.

4 Thenganatt MA, Jankovic J: The relationship between essential tremor and Parkinson's disease. Parkinsonism Relat Disord 2016;22:S162-165.

5 Diederich NJ, McIntyre DJ: Sleep disorders in Parkinson's disease: many causes, few therapeutic options. J Neurol Sci 2012;314:12-19.

6 Kleiner-Fisman G, Stern MB, Fisman DN: Health-related quality of life in Parkinson disease: correlation between Health Utilities Index III and Unified Parkinson's Disease Rating Scale (UPDRS) in U.S. male veterans. Health Qual Life Outcomes 2010;8:91.

-7 Jyothi HJ, Vidyadhara DJ, Mahadevan A, Philip M, Parmar SK, Manohari SG, Shankar SK, Raju TR, Alladi PA: Aging causes morphological alterations in astrocytes and microglia in human substantia nigra pars compacta. Neurobiol Aging 2015;36:3321-3333.

8 Liu SY, Wu JJ, Zhao J, Huang SF, Wang YX, Ge JJ, Wu P, Zuo CT, Ding ZT, Wang J: Onset-related subtypes of Parkinson's disease differ in the patterns of striatal dopaminergic dysfunction: A positron emission tomography study. Parkinsonism Relat Disord 2015;21:1448-1453.

-9 Ransmayr G: Cognitive impairment in Parkinson's disease. Psychiatr Danub 2015;27:458-461.

10 Fargel M, Grobe B, Oesterle E, Hastedt C, Rupp M: Treatment of Parkinson's disease: a survey of patients and neurologists. Clin Drug Investig 2007;27:207-218.

-11 Lithgow BJ, Shoushtarian M: Parkinson's disease: disturbed vestibular function and levodopa. J Neurol Sci 2015;353:49-58.

12 Alonso Canovas A, Luquin Piudo R, Garcia Ruiz-Espiga P, Burguera JA, Campos Arillo V, Castro A, Linazasoro G, Lopez Del Val J, Vela L, Martinez Castrillo JC: Dopaminergic agonists in Parkinson's disease. Neurologia 2014;29:230-241.

13 Talati R, Reinhart K, Baker W, White CM, Coleman CI: Pharmacologic treatment of advanced Parkinson's disease: a meta-analysis of COMT inhibitors and MAO-B inhibitors. Parkinsonism Relat Disord 2009;15:500-505.

14 Siddiqui A, Mallajosyula JK, Rane A, Andersen JK: Ability to delay neuropathological events associated with astrocytic MAO-B increase in a Parkinsonian mouse model: implications for early intervention on disease progression. Neurobiol Dis 2011;43:527-532.

15 Hao H, Shao M, An J, Chen C, Feng X, Xie S, Gu Z, Chan P, Chinese Parkinson Study G: Association of CatecholO-Methyltransferase and monoamine oxidase B gene polymorphisms with motor complications in parkinson's disease in a Chinese population. Parkinsonism Relat Disord 2014;20:1041-1045.

16 Perez-Lloret S, Rascol 0: Piribedil for the Treatment of Motor and Non-motor Symptoms of Parkinson Disease. CNS Drugs 2016;30:703-717.

17 Lemke MR: Dopamine agonists in the treatment of non-motor symptoms of Parkinson's disease: depression. Eur J Neurol 2008;15:9-14.

-18 Fox SH, Katzenschlager R, Lim SY, Ravina B, Seppi K, Coelho M, Poewe W, Rascol O, Goetz CG, Sampaio C: The Movement Disorder Society Evidence-Based Medicine Review Update: Treatments for the motor symptoms of Parkinson's disease. Mov Disord 2011;26:S2-41.

19 Pedrosa DJ, Timmermann L: Review: management of Parkinson's disease. Neuropsychiatr Dis Treat 2013;9:321-340.

20 Lebioda A, Gasche D, Dippel FW, Theobald K, Plantor S: Relevance of indirect comparisons in the German early benefit assessment and in comparison to HTA processes in England, France and Scotland. Health Econ Rev 2014;4:31.

-21 Higgins JP, Altman DG, Gotzsche PC, Juni P, Moher D, Oxman AD, Savovic J, Schulz KF, Weeks L, Sterne JA, Cochrane Bias Methods G, Cochrane Statistical Methods G: The Cochrane Collaboration's tool for assessing risk of bias in randomised trials. BMJ 2011;343:d5928.

22 Chung JH, Lee SW: Assessing the quality of randomized controlled urological trials conducted by korean medical institutions. Korean J Urol 2013;54:289-296.

-23 Chen LX, Li YL, Ning GZ, Li Y, Wu QL, Guo JX, Shi HY, Wang XB, Zhou Y, Feng SQ: Comparative efficacy and tolerability of three treatments in old people with osteoporotic vertebral compression fracture: a network meta-analysis and systematic review. PLoS One 2015;10:e0123153.

-24 Yang YJ, Baltus AE, Mathew RS, Murphy EA, Evrony GD, Gonzalez DM, Wang EP, Marshall-Walker CA, 


\section{Cellular Physiology Cell Physiol Biochem 2018;45:119-130 \begin{tabular}{ll|l} 
and Biochemistry Published online: January 15, 2018 & $\begin{array}{l}\text { (C) } 2018 \text { The Author(s). Published by S. Karger AG, Basel } \\
\text { www.karger.com/cpb }\end{array}$
\end{tabular}}

Li et al.: Efficacy of Different Drugs on PD

Barry BJ, Murn J, Tatarakis A, Mahajan MA, Samuels HH, Shi Y, Golden JA, Mahajnah M, Shenhav R, Walsh CA: Microcephaly gene links trithorax and REST/NRSF to control neural stem cell proliferation and differentiation. Cell 2012;151:1097-1112.

25 Chaimani A, Higgins JP, Mavridis D, Spyridonos P, Salanti G: Graphical tools for network meta-analysis in STATA. PLoS One 2013;8:e76654.

-26 Salanti G, Ades AE, Ioannidis JP: Graphical methods and numerical summaries for presenting results from multiple-treatment meta-analysis: an overview and tutorial. J Clin Epidemiol 2011;64:163-171.

27 Smith KM, Eyal E, Weintraub D, Investigators A: Combined rasagiline and antidepressant use in Parkinson disease in the ADAGIO study: effects on nonmotor symptoms and tolerability. JAMA Neurol 2015;72:88-95.

28 Nomoto M, Mizuno Y, Kondo T, Hasegawa K, Murata M, Takeuchi M, Ikeda J, Tomida T, Hattori N: Transdermal rotigotine in advanced Parkinson's disease: a randomized, double-blind, placebo-controlled trial. J Neurol 2014;261:1887-1893.

29 Nicholas AP, Borgohain R, Chana P, Surmann E, Thompson EL, Bauer L, Whitesides J, Elmer LW, Investigators SPS: A randomized study of rotigotine dose response on 'off' time in advanced Parkinson's disease. J Parkinsons Dis 2014;4:361-373.

-30 Ray Chaudhuri K, Martinez-Martin P, Antonini A, Brown RG, Friedman JH, Onofrj M, Surmann E, Ghys L, Trenkwalder C: Rotigotine and specific non-motor symptoms of Parkinson's disease: post hoc analysis of RECOVER. Parkinsonism Relat Disord 2013;19:660-665.

-31 Mizuno Y, Nomoto M, Kondo T, Hasegawa K, Murata M, Takeuchi M, Ikeda J, Tomida T, Hattori N, Rotigotine Trial G: Transdermal rotigotine in early stage Parkinson's disease: a randomized, double-blind, placebocontrolled trial. Mov Disord 2013;28:1447-1450.

-32 Grosset KA, Malek N, Morgan F, Grosset DG: Inhaled dry powder apomorphine (VR040) for 'off ' periods in Parkinson's disease: an in-clinic double-blind dose ranging study. Acta Neurol Scand 2013;128:166-171.

-33 Grosset KA, Malek N, Morgan F, Grosset DG: Inhaled apomorphine in patients with 'on-off' fluctuations: a randomized, double-blind, placebo-controlled, clinic and home based, parallel-group study. J Parkinsons Dis 2013;3:31-37.

-34 Stocchi F, Rabey JM: Effect of rasagiline as adjunct therapy to levodopa on severity of OFF in Parkinson's disease. Eur J Neurol 2011;18:1373-1378.

-35 Watts RL, Lyons KE, Pahwa R, Sethi K, Stern M, Hauser RA, Olanow W, Gray AM, Adams B, Earl NL, Study I: Onset of dyskinesia with adjunct ropinirole prolonged-release or additional levodopa in early Parkinson's disease. Mov Disord 2010;25:858-866.

-36 Hauser RA, Schapira AH, Rascol O, Barone P, Mizuno Y, Salin L, Haaksma M, Juhel N, Poewe W: Randomized, double-blind, multicenter evaluation of pramipexole extended release once daily in early Parkinson's disease. Mov Disord 2010;25:2542-2549.

37 Singer C, Lamb J, Ellis A, Layton G, Sumanirole for Early Parkinson's Disease Study G: A comparison of sumanirole versus placebo or ropinirole for the treatment of patients with early Parkinson's disease. Mov Disord 2007;22:476-482.

-38 Mizuno Y, Abe T, Hasegawa K, Kuno S, Kondo T, Yamamoto M, Nakashima M, Kanazawa I, Group SS: Ropinirole is effective on motor function when used as an adjunct to levodopa in Parkinson's disease: STRONG study. Mov Disord 2007;22:1860-1865.

-39 Jankovic J, Watts RL, Martin W, Boroojerdi B: Transdermal rotigotine: double-blind, placebo-controlled trial in Parkinson disease. Arch Neurol 2007;64:676-682.

-40 Castro-Caldas A, Delwaide P, Jost W, Merello M, Williams A, Lamberti P, Aguilar M, Del Signore S, Cesaro P, Parkinson-Control Study G: The Parkinson-Control study: a 1-year randomized, double-blind trial comparing piribedil (150 mg/day) with bromocriptine (25 mg/day) in early combination with levodopa in Parkinson's disease. Mov Disord 2006;21:500-509.

41 Reichmann H, Boas J, Macmahon D, Myllyla V, Hakala A, Reinikainen K, ComQol Study G: Efficacy of combining levodopa with entacapone on quality of life and activities of daily living in patients experiencing wearing-off type fluctuations. Acta Neurol Scand 2005;111:21-28.

42 Rascol O, Brooks DJ, Melamed E, Oertel W, Poewe W, Stocchi F, Tolosa E, group Ls: Rasagiline as an adjunct to levodopa in patients with Parkinson's disease and motor fluctuations (LARGO, Lasting effect in Adjunct therapy with Rasagiline Given Once daily, study): a randomised, double-blind, parallel-group trial. Lancet 2005;365:947-954.

43 Wong KS, Lu CS, Shan DE, Yang CC, Tsoi TH, Mok V: Efficacy, safety, and tolerability of pramipexole in untreated and levodopa-treated patients with Parkinson's disease. J Neurol Sci 2003;216:81-87.

$\$ 44$ Im JH, Ha JH, Cho IS, Lee MC: Ropinirole as an adjunct to levodopa in the treatment of Parkinson's disease: a 16-week bromocriptine controlled study. J Neurol 2003;250:90-96.

45 Brooks DJ, Sagar H, Group UK-IES: Entacapone is beneficial in both fluctuating and non-fluctuating patients with Parkinson's disease: a randomised, placebo controlled, double blind, six month study. J Neurol 


\section{Cellular Physiology Cell Physiol Biochem 2018;45:119-130 \begin{tabular}{l|l|l} 
and Biochemistry 10.1159/000486252 & $\begin{array}{l}\text { C } 2018 \text { The Author(s). Published by S. Karger AG, Basel } \\
\text { www.karger.com/cpb }\end{array}$ \\
\hline
\end{tabular}}

Li et al.: Efficacy of Different Drugs on PD

Neurosurg Psychiatry 2003;74:1071-1079.

-46 Pogarell 0, Gasser T, van Hilten JJ, Spieker S, Pollentier S, Meier D, Oertel WH: Pramipexole in patients with Parkinson's disease and marked drug resistant tremor: a randomised, double blind, placebo controlled multicentre study. J Neurol Neurosurg Psychiatry 2002;72:713-720.

47 Rascol O, Brooks DJ, Korczyn AD, De Deyn PP, Clarke CE, Lang AE: A five-year study of the incidence of dyskinesia in patients with early Parkinson's disease who were treated with ropinirole or levodopa. N Engl J Med 2000;342:1484-1491.

48 Maranis S, Tsouli S, Konitsiotis S: Treatment of motor symptoms in advanced Parkinson's disease: a practical approach. Prog Neuropsychopharmacol Biol Psychiatry 2011;35:1795-1807.

49 Akl H, Vervloessem T, Kiviluoto S, Bittremieux M, Parys JB, De Smedt H, Bultynck G: A dual role for the anti-apoptotic Bcl-2 protein in cancer: mitochondria versus endoplasmic reticulum. Biochim Biophys Acta 2014;1843:2240-2252.

-50 Dehay B, Bourdenx M, Gorry P, Przedborski S, Vila M, Hunot S, Singleton A, Olanow CW, Merchant KM, Bezard E, Petsko GA, Meissner WG: Targeting alpha-synuclein for treatment of Parkinson's disease: mechanistic and therapeutic considerations. Lancet Neurol 2015;14:855-866.

51 Montoya A, Lal S, Menear M, Duplessis E, Thavundayil J, Schmitz N, Lepage M: Apomorphine effects on episodic memory in young healthy volunteers. Neuropsychologia 2008;46:292-300.

-52 Martinez-Martin P, Reddy P, Antonini A, Henriksen T, Katzenschlager R, Odin P, Todorova A, Naidu Y, Tluk S, Chandiramani C, Martin A, Chaudhuri KR: Chronic subcutaneous infusion therapy with apomorphine in advanced Parkinson's disease compared to conventional therapy: a real life study of non motor effect. J Parkinsons Dis 2011;1:197-203.

53 Campbell JC, Jeyamohan SB, De La Cruz P, Chen N, Shin D, Pilitsis JG: Place conditioning to apomorphine in rat models of Parkinson's disease: differences by dose and side-effect expression. Behav Brain Res 2014;275:114-119.

54 Pan J, Zhao YX, Wang ZQ Jin L, Sun ZK, Chen SD: Expression of FasL and its interaction with Fas are mediated by c-Jun $\mathrm{N}$-terminal kinase (JNK) pathway in 6-OHDA-induced rat model of Parkinson disease. Neurosci Lett 2007;428:82-87.

-55 Trenkwalder C, Chaudhuri KR, Garcia Ruiz PJ, LeWitt P, Katzenschlager R, Sixel-Doring F, Henriksen T, Sesar A, Poewe W, Expert Consensus Group for Use of Apomorphine in Parkinson's D, Baker M, CeballosBaumann A, Deuschl G, Drapier S, Ebersbach G, Evans A, Fernandez H, Isaacson S, van Laar T, Lees A, Lewis S, Martinez Castrillo JC, Martinez-Martin P, Odin P, O’Sullivan J, Tagaris G, Wenzel K: Expert Consensus Group report on the use of apomorphine in the treatment of Parkinson's disease--Clinical practice recommendations. Parkinsonism Relat Disord 2015;21:1023-1030.

-56 Lorenc-Koci E, Lenda T, Antkiewicz-Michaluk L, Wardas J, Domin H, Smialowska M, Konieczny J: Different effects of intranigral and intrastriatal administration of the proteasome inhibitor lactacystin on typical neurochemical and histological markers of Parkinson's disease in rats. Neurochem Int 2011;58:839-849.

57 Henriksen T: Clinical insights into use of apomorphine in Parkinson's disease: tools for clinicians. Neurodegener Dis Manag 2014;4:271-282.

58 de Leeuw van Weenen JE, Parlevliet ET, Schroder-van der Elst JP, van den Berg SA, Willems van Dijk K, Romijn JA, Pijl H: Pharmacological modulation of dopamine receptor D2-mediated transmission alters the metabolic phenotype of diet induced obese and diet resistant C57Bl6 mice. Exp Diabetes Res 2011;2011:928523.

59 Oda T, Kume T, Izumi Y, Takada-Takatori Y, Niidome T, Akaike A: Bromocriptine, a dopamine D(2) receptor agonist with the structure of the amino acid ergot alkaloids, induces neurite outgrowth in PC12 cells. Eur J Pharmacol 2008;598:27-31.

60 Liu Y, Liao S, Quan H, Lin Y, Li J, Yang Q: Involvement of microRNA-135a-5p in the Protective Effects of Hydrogen Sulfide Against Parkinson's Disease. Cell Physiol Biochem 2016;40:18-26.

61 Mills EJ, Thorlund K, Ioannidis JP: Demystifying trial networks and network meta-analysis. BMJ 2013;346:f2914.

62 Movement Disorder Society Task Force on Rating Scales for Parkinson's D: The Unified Parkinson's Disease Rating Scale (UPDRS): status and recommendations. Mov Disord 2003;18:738-750.

63 Goetz CG, Tilley BC, Shaftman SR, Stebbins GT, Fahn S, Martinez-Martin P, Poewe W, Sampaio C, Stern MB, Dodel R, Dubois B, Holloway R, Jankovic J, Kulisevsky J, Lang AE, Lees A, Leurgans S, LeWitt PA, Nyenhuis D, Olanow CW, Rascol O, Schrag A, Teresi JA, van Hilten JJ, LaPelle N, Movement Disorder Society URTF: Movement Disorder Society-sponsored revision of the Unified Parkinson's Disease Rating Scale (MDSUPDRS): scale presentation and clinimetric testing results. Mov Disord 2008;23:2129-2170.

64 Verbaan D, van Rooden SM, Benit CP, van Zwet EW, Marinus J, van Hilten JJ: SPES/SCOPA and MDS-UPDRS: formulas for converting scores of two motor scales in Parkinson's disease. Parkinsonism Relat Disord 2011;17:632-634. 